\title{
On the Formation of Boxy and Disky Elliptical Galaxies
}

\section{Citation}

Naab, Thorsten, Andreas Burkert, and Lars Hernquist. 1999. "On the Formation of Boxy and Disky Elliptical Galaxies." The Astrophysical Journal 523 (2): L133-36. https:// doi.org/10.1086/312275.

\section{Permanent link}

http://nrs.harvard.edu/urn-3:HUL.InstRepos:41381670

\section{Terms of Use}

This article was downloaded from Harvard University's DASH repository, and is made available under the terms and conditions applicable to Other Posted Material, as set forth at http:// nrs.harvard.edu/urn-3:HUL.InstRepos:dash.current.terms-of-use\#LAA

\section{Share Your Story}

The Harvard community has made this article openly available.

Please share how this access benefits you. Submit a story.

Accessibility 


\title{
On the Formation of Boxy and Disky Elliptical Galaxies
}

\author{
Thorsten Naab \& Andreas Burkert \\ Max-Planck-Institut für Astronomie, Königstuhl 17, \\ D-69117 Heidelberg, \\ Germany \\ Lars Hernquist \\ Center for Astrophysics, 60 Garden Street, \\ Cambridge, MA 02138, \\ USA
}

Received — 


\begin{abstract}
The origin of boxy and disky elliptical galaxies is investigated. The results of two collisionless N-body simulations of spiral-spiral mergers with mass ratios of 1:1 and 3:1 are discussed and the projected properties of the merger remnants are investigated. It is shown that the equal-mass merger leads to an anisotropic, slowly rotating system with preferentially boxy isophotes and significant minor axis rotation. The unequal-mass merger results in the formation of a rotationally supported elliptical with disky isophotes and small minor axis rotation. The observed scatter in the kinematical and isophotal properties of both classes of elliptical galaxies can be explained by projection effects.
\end{abstract}

Subject headings: galaxies: interaction-galaxies: structure - galaxies: evolution - methods: numerical 


\section{Introduction}

Elliptical galaxies have long been considered as old, coeval systems, consisting of a dynamically relaxed, spheroidal stellar population with a universal $r^{1 / 4}$ surface brightness profile. More detailed observations have however shown that these systems can be subdivided into two classes with distinct kinematical and orbital properties. Low-luminosity ellipticals are isotropic and rotationally supported, with small minor axis rotation and disky deviations of their isophotal shapes from perfect ellipses Bender 1988; Bender, Döbereiner \& Möllenhoff 1988, hereafter BDM; Kormendy \& Bender 1996 and references therein). High-luminosity ellipticals, on the other hand, are anisotropic, slow rotators with large minor axis rotation, boxy isophotes and, occasionally, with kinematically distinct cores. Bender el al. (1989) demonstrated that both groups have different radio and X-ray luminosities and recent high-resolution observations exhibit that disky ellipticals have steep power-law cores in contrast to boxy ellipticals with flat cores and central density cusps (Lauer et al. 1995, Faber et al. 1997).

On the theoretical side, Toomre \& Toomre (1972) proposed that early type galaxies originate from major mergers of disk galaxies. This "merger hypothesis" has been investigated in great details by numerous authors using numerical simulations (see Barnes \& Hernquist 1992 for a review). The first fully self-consistent merger models of two equal-mass rotationally supported stellar disks, embedded in dark matter halos were performed by Barnes (1988) and Hernquist (1992). They found that mergers indeed lead to a slowly rotating, pressure supported, anisotropic spheroidal system. In the inner regions, the remnants were however too diffuse, leading to strong deviations from the observed de Vaucouleurs profiles which requires an inner $r^{-1}$ density profile. This result can be explained by phase space limitations (Carlberg 1986). Subsequent investigations by Hernquist et al. (1993) showed that mergers of progenitors with massive bulge components could resolve this problem, leading to ellipticals with small core radii and surface brightness profiles that are in excellent agreement with observations. Hernquist (1993b) and subsequently Heyl, Hernquist \& Spergel (1994) and Steinmetz \& Buchner (1995) noted departures from pure ellipses in their equal-mass merger remnants. The same remnant when viewed from different orientations appeared either boxy or disky. This seems to be in contradiction with the observations. As disky and boxy ellipticals have different radio and X-ray properties that should not depend on viewing angle their isophotal shapes cannot change as a result of projection effects. In agreement with observations of boxy ellipticals, Barnes(1992) and Heyl et al. (1996) also found misalignments between the spin and minor axis in major merger remnants which however seemed to be larger than observed (Franx Illingworth \& De Zeeuw 1991). 
It has been argued by Kormendy \& Bender (1996) and Faber et al. (1997) that gaseous mergers lead to distinct inner gaseous disks in the merger remnants which subsequently turn into stars, generating disky isophotes. In contrast, boxy ellipticals would form from purely dissipationless mergers. This idea has been addressed in details by Bekki \& Shioya (1997) and Bekki (1998). Bekki \& Shioya (1997) simulated mergers including gaseous dissipation and star formation. They found that the rapidity of gas consumption affects the isophotal shapes. Secular star formation however leads to final density profiles which deviate significantly from the observed $\mathrm{r}^{1 / 4}$-profiles in radial regimes where all ellipticals show almost perfect de Vaucouleurs laws (Burkert 1993). These calculations and models by Mihos \& Hernquist (1996) demonstrate that the effect of gas and star formation changes the structure of merger remnants as such a dissipative component would most likely lead to strong deviations from the $\mathrm{r}^{1 / 4}$-profiles which seems to be a result of dissipationless, violent relaxation processes. Nevertheless the observations of metal enhanced, decoupled and rapidly spinning disk-like cores (Bender \& Surma 1992; Davies Sadler \& Peletier 1993; Bender \& Davies 1996) shows that even in boxy ellipticals gas must have present. Numerical simulations show, that these features would result naturally from gas infall during the merger process Barnes \& Hernquist 1996; Mihos \& Hernquist 1996). The influence of gas on the global structure of elliptical galaxies is not well understood as it is sensitive to uncertain details about star formation (Barnes \& Hernquist 1996).

Recently Barnes (1998) proposed a scenario of the origin of rapidly rotating ellipticals that does not require gaseous dissipation. He showed that such systems would result from the merger of a large disk galaxy with a smaller companion. In addition, the edge on view shows a disky morphology. Taking this model into account, boxy and disky ellipticals should result from equal- and unequal-mass mergers, respectively.

In this letter the Barnes hypothesis is investigated in greater details. We present the results of two merger simulations, a 1:1 merger and a 3:1 merger. The definition of the global orbital and kinematical properties depends strongly on the method used. We therefore apply the same data reduction method to our model galaxies which is used to derive the global parameters of observed ellipticals and with which we compare our results. The equal-mass merger indeed leads to a boxy elliptical whereas the unequal-mass merger forms a disky elliptical, with detailed kinematical properties that are in perfect agreement with the observations. 


\section{The merger models}

The spiral galaxies are constructed in dynamical equilibrium using the method described by Hernquist (1993a). We use the following system of units: gravitational constant $G=1$, exponential scale length of the larger disk $\mathrm{h}=1$ and mass of the larger disk $M_{d}=1$. Each galaxy consists of an exponential disk, a spherical, non-rotating bulge with mass $M_{b}=1 / 3$, a Hernquist density profile (Hernquist 1990) and a scale length $r_{b}=0.2 h$ and a spherical pseudo-isothermal halo with a mass $M_{d}=5.8$, cut-off radius $r_{c}=10 h$ and core radius $\gamma=1 h$.

The N-body simulations were performed using a direct summation code with the special purpose hardware GRAPE (Sugimoto et al. 1990). The 1:1 merger was calculated adopting in total 400000 particles with each galaxy consisting of 20000 bulge particles, 60000 disk particles and 120000 halo particles. For the 3:1 merger the parameters of the more massive galaxy were as described above. The low-mass galaxy contained $1 / 3$ the mass and number of particles in each component, with a disk scale length of $h=\sqrt{1 / 3}$, as expected from the Tully-Fisher relation. For the gravitational softening we used a value of $\epsilon=0.07$. In agreement with Walker et al. (1996) we noticed a growing bar mode in the disk for test cases where we evolved galaxies in isolation. This effect is however reduced considerably with respect to previous calculations, due to our choice of twice as many halo particles than disk particles.

For both mergers, the galaxies approach each other on nearly parabolic orbits with an initial separation of 30 length units and a pericenter distance of 2 length units. The inclinations of the two disks relative to the orbit plane are $t_{1}=30^{\circ}$ and $t_{2}=-30^{\circ}$ with arguments of pericenter of $\omega_{1}=30^{\circ}$ and $\omega_{2}=-30^{\circ}$. These values are most likely for random encounters. In both simulations the merger remnants were allowed to settle into equilibrium for approximately 10 dynamical times after the merger was complete. Then their equilibrium state was analysed.

\section{Analysis of the equilibrium states}

In order to compare the simulation with observations we follow as closely as possible the analysis used by BDM. An artificial image of the remnant is created by binning the central 10 length units into $128 \times 128$ pixels. This picture is smoothed with a Gaussian filter of standard deviation 1.5 pixels. The isophotes and their deviations from perfect ellipses are then determined using a reduction package kindly provided by Ralf Bender. 
Figure 1 shows the radial distribution of $a_{4}$ along the major axis for 200 random projections. There is a clear trend for the 1:1 merger to have boxy (negative $a_{4}$ ) isophotes while the 3:1 merger has disky deviations (positive $a_{4}$ ) inside one half-mass radius. Following the definition of BDM for the global properties of observed elliptical galaxies, we determine for every projection $a_{4}$ as the mean value between $0.25 r_{e}$ and $1.0 r_{e}$, with $r_{e}$ being the spherical half light radius. In case of a strong peak in the $a_{4}$-distribution with an absolute value that is larger than the absolute mean value, we choose the peak value. In Figure 2 representative isophotal contours of the simulation of a boxy (1:1 merger) and a disky (3:1 merger) merger remnant are shown.

The characteristic ellipticity $\epsilon$ is defined as the isophotal ellipticity at $1.5 r_{e}$. The central velocity dispersion $\sigma$ is determined as the average projected velocity dispersion of the stars inside a projected galactocentric distance of $0.2 r_{e}$. Finally, we define the characteristic rotational velocity along the major and the minor axis as the projected rotational velocity determined around $1.5 r_{e}$ and $0.5 r_{e}$, respectively.

Figure 3 shows the characteristic isophotal and kinematical properties of the 1:1 merger (filled circles) and of the 3:1 merger (open circles) for 200 random projections. One can clearly see that the 1:1 merger produces remnants that are anisotropic and boxy with large minor axis rotation whereas the 3:1 merger forms an isotropic and disky elliptical with small minor axis rotation. These results are in excellent agreement with the observations of BDM.

\section{Discussion and Conclusions}

Our calculations demonstrate that the observed dichotomy between boxy and disky ellipticals could originate from variations in the mass ratios of the merger components. In general, the isophotal shapes change with radius. An analysis applying methods and definitions similar to those used for observed ellipticals does however lead to a clear separation of properties between the models of equal and unequal-mass mergers in agreement with the observed structure of boxy and disky ellipticals, respectively. A trend for equal-mass mergers to form preferentially boxy ellipticals has already been noted before by Steinmetz \& Buchner (1995), who did however neglect the bulge component. We find that a 3:1 merger is still efficient enough in order to disrupt the disks, leading to spheroidal galaxies with de Vaucouleurs

profiles. Our analysis also shows that these systems are rotationally supported with disky isophotes in the region inside one effective radius. 
Projection effects do not change the fundamental difference between equal and unequal-mass merger remnants. They do however lead to a large spread in the global parameters. This is in very good agreement with the observed parameter distribution (BDM; Bender et al. 1989).

In contradiction to the common believe that disky E/S0 galaxies are formed involving dissipative processes like star formation (Bekki \& Shioya 1997) we conclude that pure stellar mergers can in principle explain the observed dichotomy between disky and boxy ellipticals. This result is supported by additional merger simulations with varying mass ratios and orbital parameters which will be discussed in more details in a subsequent paper.

Observations show that disky ellipticals have on average lower luminosities than boxy ellipticals (BDM). Our results would indicate that low-mass elliptical galaxies preferentially formed by unequal-mass mergers of disk-galaxies whereas equal-mass mergers dominated the formation of high-mass ellipticals. This result is puzzeling as there does not exist a convincing argument for why low mass ellipticals should have suffered mainly minor mergers while high mass ones should have evolved mainly through major mergers.

Since dissipative features are observed in all types of elliptical galaxies in can not generally be ruled out, that the gas dynamics and star formation have played an important role for the formation of boxy and disky ellipticals.

We thank Ralf Bender, Hans-Walter Rix and Ralf Klessen for helpful disussions. 


\section{REFERENCES}

Barnes, J. E. 1988, ApJ, 331, 699

Barnes, J. E. 1992, ApJ, 393, 484

Barnes, J. E. \& Hernquist, L. 1992, ARA\&A, 30, 705

Barnes, J. E. \& Hernquist, L. 1996, ApJ, 471, 115

Barnes, J., Galaxies: interactions and induced star formation: lecture notes 1996 / Saas Fee Advanced Course 26, eds. D. Friedli, L. Martinet, and D. Pfenniger, Springer, 1998

Bender, R. 1988, A\&A, 193, L7

Bender, R., Doebereiner, S. \& Möllenhoff, C. 1988, A\&AS, 74, 385

Bender, R., Surma, P., Döbereiner, S., Möllenhoff, C. \& Madejsky, R. 1989, A\&A, 217, 35

Bender, R. \& Surma, P. 1992, A\&A, 258, 250

Bender, R. \& Davies, R. L. 1996, IAU Symposia, 171,

Burkert, A. 1993, A\&A, 278, 23

Bekki, K. \& Shioya, Y. 1997, ApJ, 478, L17

Bekki, K. 1998, ApJ, 502, L133

Carlberg, R. G. 1986, ApJ, 310, 593

Davies, R. L., Sadler, E. M. \& Peletier, R. F. 1993, MNRAS, 262, 650

Faber, S. M., et al. 1997, AJ, 114, 1771

Franx, M. , Illingworth, G. \& De Zeeuw, T. 1991, ApJ, 383, 112

Hernquist, L. 1990, ApJ, 356, 359

Hernquist, L. 1992, ApJ, 400, 460

Hernquist, L. 1993a, ApJS, 86, 389 
Hernquist, L. 1993b, ApJ, 409, 548

Hernquist, L. , Spergel, D. N. \& Heyl, J. S. 1993, ApJ, 416, 415

Heyl, J. S., Hernquist, L. \& Spergel, D. N. 1994, ApJ, 427, 165

Heyl, J. S., Hernquist, L. \& Spergel, D. N. 1996, ApJ, 463, 69

Kormendy, J. \& Bender, R. 1996, ApJ, 464, L119

Lauer, T. R., et al. 1995, AJ, 110, 2622

Mihos, J. C. \& Hernquist, L. 1996, ApJ, 464, 641

Steinmetz, M. \& Buchner, S. 1995, Galaxies in the young universe, proceedings of a workshop held at Ringberg Castle, eds. H. Hippelein, K. Meisenheimer \& H.-J. Röser, Springer, p. 215

Sugimoto, D., Chikada, Y., Makino, J., Ito, T., Ebisuzaki, T. \& Umemura, M. 1990, Nature, 345, 33

Toomre, A. \& Toomre, J. 1972, ApJ, 178, 623

Walker, I. R., Mihos, J. C. \& Hernquist, L. 1996, ApJ, 460, 121 


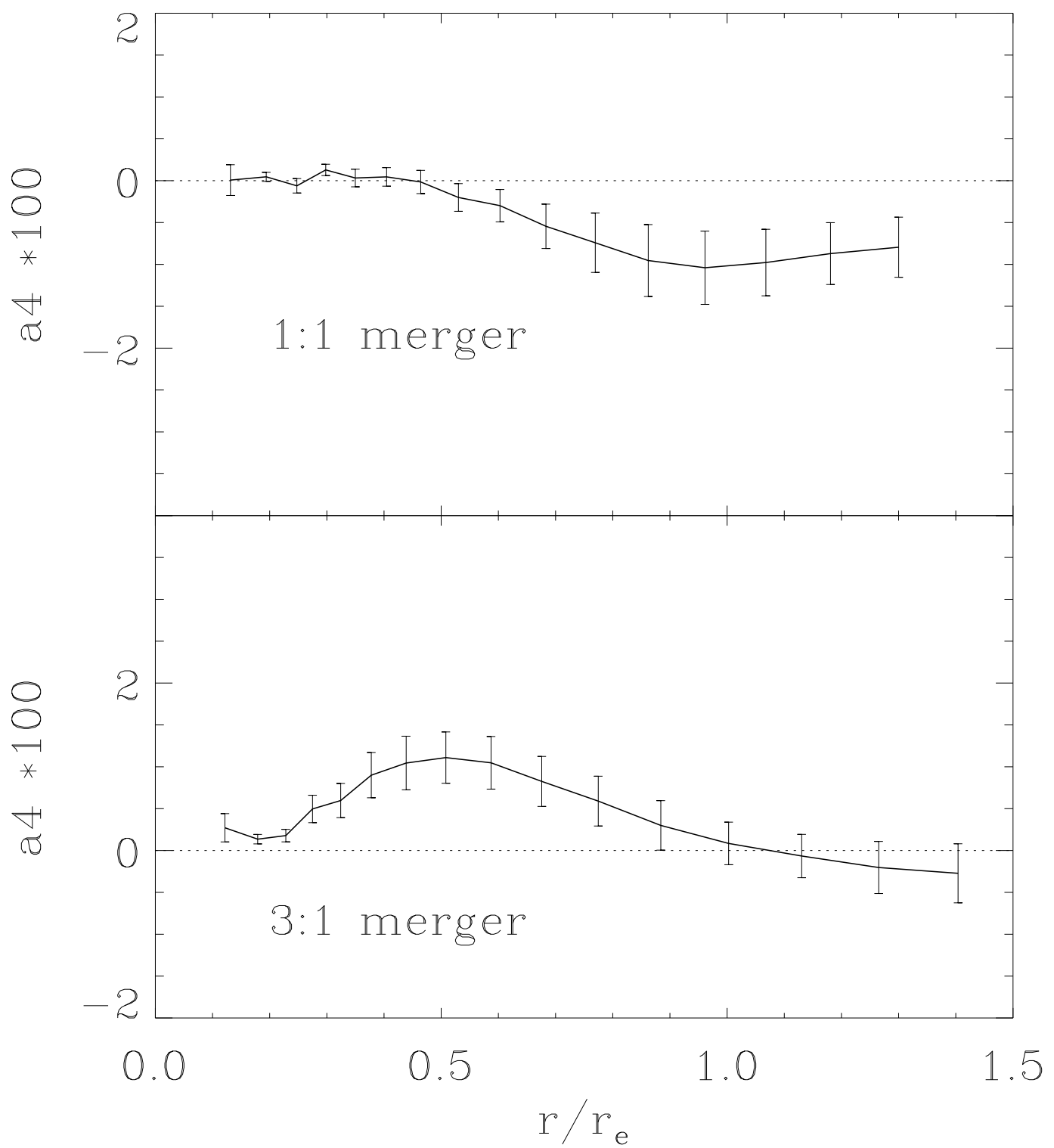

Fig. 1.- Mean variation of $a_{4}$ along the apparent radius for 200 random projections with $1 \sigma$ error bars which indicate the variation due to projection effects. The 1:1 merger leads to a boxy isophotes (upper panel), while the 3:1 has positive values of $a_{4}$ (disky isophotes) inside one half light radius $r_{e}$. 

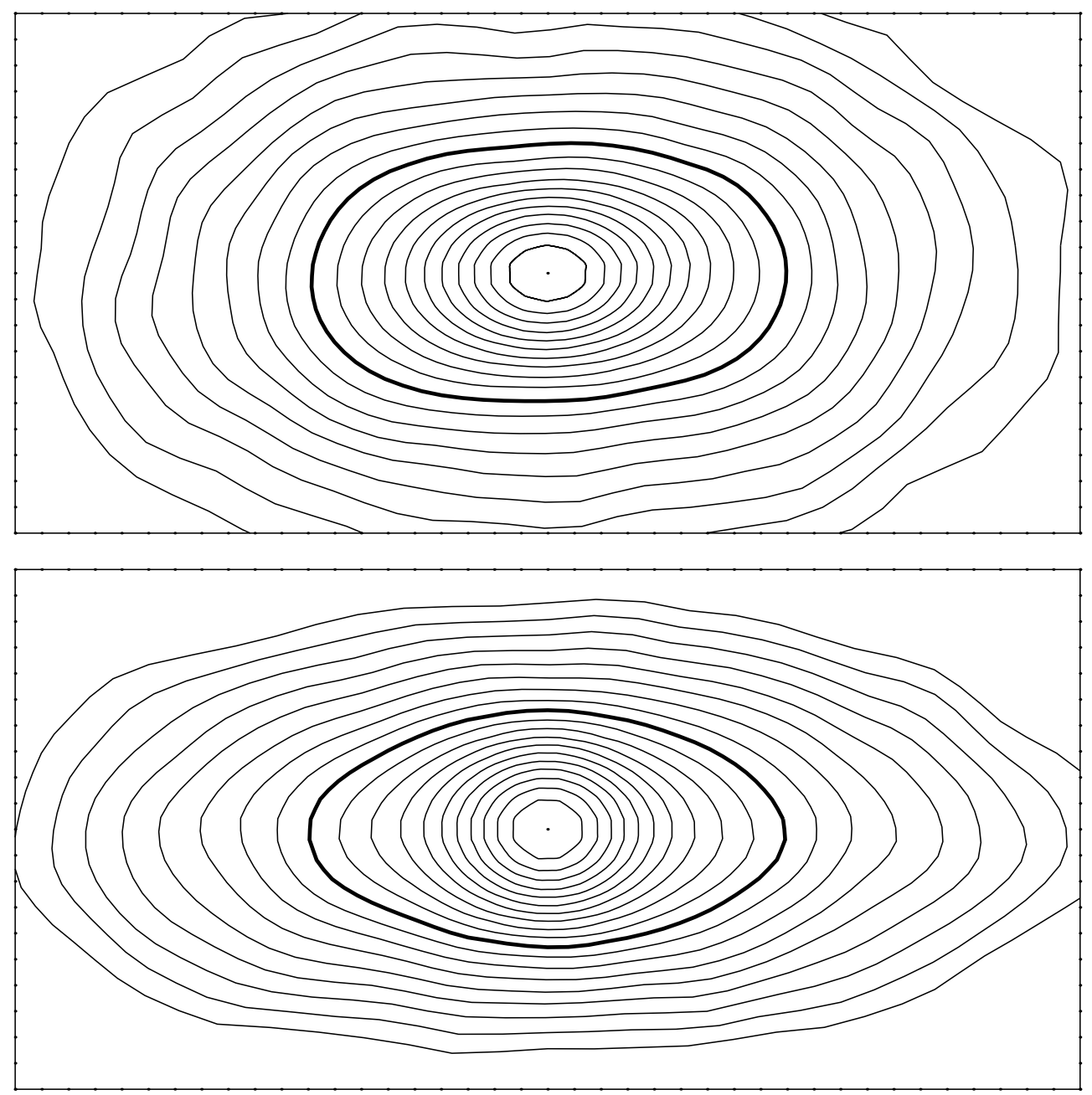

Fig. 2.- Representative isophotal contours of a simulated 1:1 merger remnant with boxy isophotes (upper panel) and a 3:1 merger remnant with disky isophotes (lower panel). The thick contour indicates the region where $a 4$ was determined. 

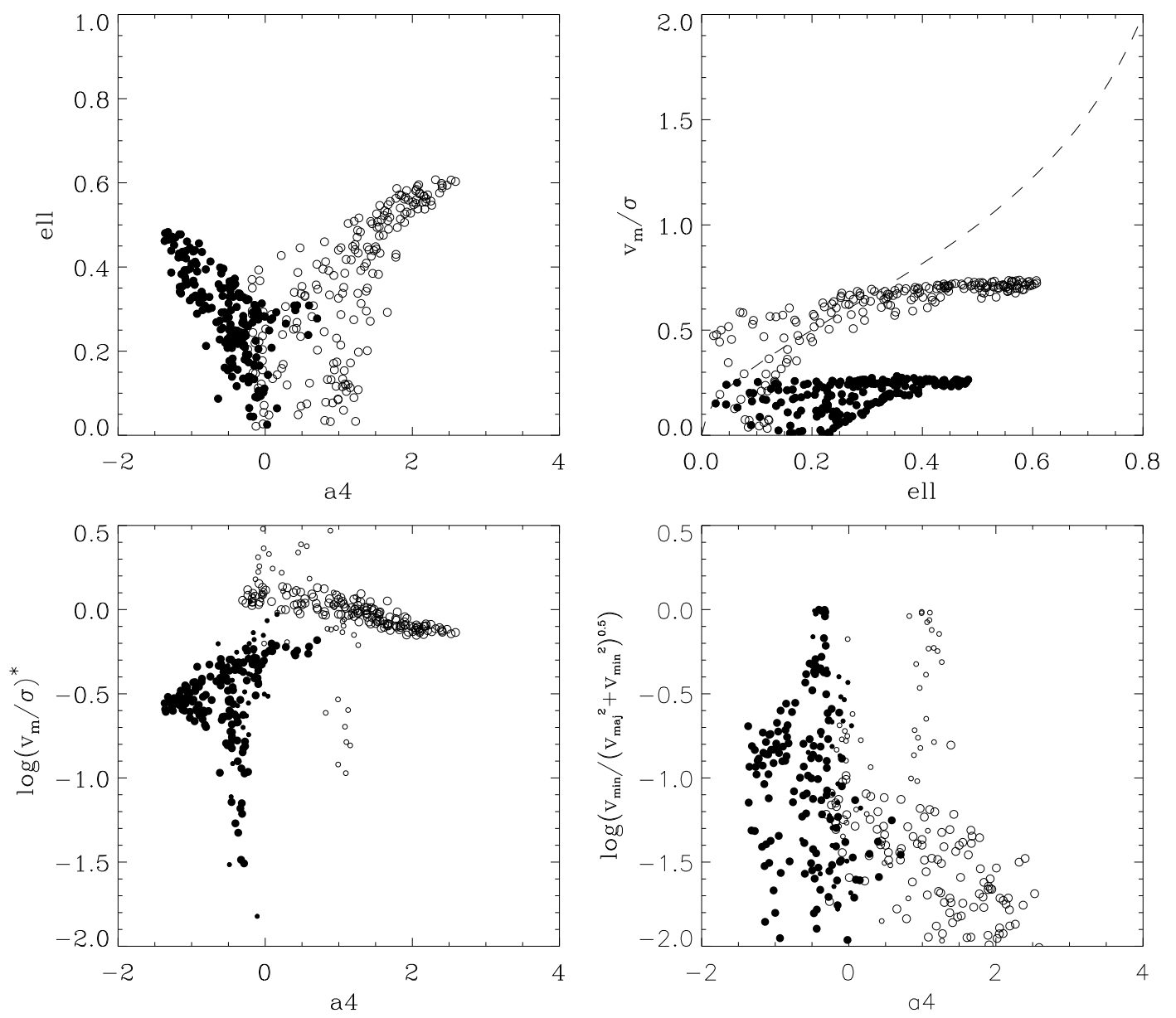

Fig. 3.- Kinematical and photometric properties for 200 random projections of the merger remnants. Filled circles show the values for the equal-mass merger while open circles show the values for the 3:1 merger. Upper left panel: Ellipticity of the remnants versus $a_{4}$. Upper right panel: Rotational velocity over central velocity dispersion versus ellipticity. The dashed line shows the theoretical curve for an oblate isotropic rotator. Lower left panel: Anisotropy parameter $(v / \sigma)^{*}$ versus $a_{4}$. The small dots and circles indicate projections with apparent ellipticities smaller than 0.2. For such values the determination of the major axis is very uncertain, leading to large errors. Lower left panel: Amount of minor axis rotation versus $a_{4}$ with $v_{m a j}$ an $v_{\min }$ being the maximum velocity along the major and minor axis, respectively. 